\title{
CUMULATIVE WEIGHTING OPTIMIZATION: THE DISCRETE CASE
}

\author{
Kin Lin \\ Steven I. Marcus \\ Department of Electrical \& Computer Engineering \\ Institute for Systems Research \\ University of Maryland \\ College Park, MD 20742, USA
}

\begin{abstract}
Global optimization problems are relevant in many fields (e.g., control systems, operations research, economics). There are many approaches to solving these problems. One particular approach is modelbased methods, which are a class of random search methods. A model-based method iteratively updates its probability density function. At each step, additional weight is given to solution subspaces that are more likely to yield an optimal objective value. Model-based methods can be analyzed by writing down a corresponding system of differential equations similar to the well known Fokker-Planck equation, which models the evolution of probability density functions for diffusions. We propose an innovative model-based method, Cumulative Weighting Optimization (CWO), which can be proven to converge to an optimal solution. Using this rigorous theoretical foundation, we design a CWO-based numerical algorithm for solving global optimization problems. Interestingly, the well-known cross-entropy (CE) method is a special case of this CWO-based algorithm.
\end{abstract}

\section{INTRODUCTION}

Many problems in engineering and science can be formulated as global optimization problems. These problems are challenging when their objective functions are nonlinear (e.g., non-convex, multi-modal, or badly scaled). If we are only interested in finding their local extrema and they are differentiable, then the standard local optimization method (i.e., first derivative being zero) would suffice. If there are only a few local extrema, then we can easily find a global optimal solution by evaluating all of them. However, this approach does not work on objective functions with absence of structural information (e.g., nondifferentiable), or in the presence of many local extrema. Approaches developed to solve these problems can be divided into two categories: deterministic and random search algorithms. Random search algorithms can be further divided into instance-based (e.g., simulated annealing, genetic algorithm, tabu search, nested partitions, generalized hill climbing, and evolutionary programming) and model-based algorithms (e.g., annealing-adaptive search, cross-entropy (CE), and estimation of distribution algorithms (EDAs)). For the interested reader, Hu et al. (2012) have a recent survey paper on model-based methods, which also contains references to instance-based methods mentioned in this paragraph.

We provide a new addition, inspired by Cumulative Prospect Theory (CPT), to the class of model-based methods. The new CWO-based algorithms have an intuitive connection with the risk-sensitive nature of the human decision making process.

In the rest of this paper, we will proceed in the following sequence. In Section 2, we present our problem statement. In Section 3, we introduce probability weighting functions. In Section 4, we will work with the case when the solution space is discrete (i.e., $\mathscr{X}=\{1,2,3,4, \ldots\}$ ) and provide the reader some insight into the construction of our probability weight updating equation. Later in the same section, we 


\section{Lin and Marcus}

will prove the convergence properties for the equation. Our convergence proof is a modified version of the model-based evolutionary optimization by Wang et al. (2010) and Wang (2011), which are a recent addition to model-based approaches. Finally, in Section 5, we will suggest a CWO-based algorithm and present its simulation results. Other CWO-based algorithms will be explored in the future.

\section{PROBLEM}

In many engineering applications, we are looking for a "best" solution based on some criterion. For example, in the well known traveling salesman problem (TSP), we are looking for the cheapest route that visits all cities and terminates at the starting point. Problems of this nature can be formulated as the following optimization problem:

$$
x^{*} \in \arg \max _{x \in \mathscr{X}} H(x),
$$

where $x^{*}$ is an optimal solution to the problem and $\mathscr{X}$ is the solution space (in many applications $\mathscr{X} \subset \mathscr{R}^{n}$ ). $H: \mathscr{X} \rightarrow \mathscr{R}$, the objective function, is a bounded deterministic measurable function. In the rest of this paper we assume the following:

Assumption 1 There exists a global optimal solution to Equation (1), i.e., $\exists x^{*} \in \mathscr{X}$ such that $H(x) \leq$ $H\left(x^{*}\right) \forall x \neq x^{*} \forall x \in \mathscr{X}$.

This assumption is true for many optimization problems. For example, the assumption holds trivially when $\mathscr{X}$ is a finite discrete solution space. We do not assume any other structural information about the objective function (i.e., convexity, differentiability). The objective function may have many local extrema.

It is common in many situations to introduce a measurable strictly increasing fitness function, $\phi: \mathscr{R} \rightarrow$ $\mathscr{R}^{+}$, and reformulate Equation (1) as:

$$
x^{*} \in \arg \max _{x \in \mathscr{X}} \phi(H(x)) .
$$

Since the reformulated problem guarantees the range of the new fitness-objective function (i.e., $\phi(H(\cdot)))$ will always be non-negative, and it is equivalent to the original problem, we will solve Equation (2) in place of Equation (1).

\section{PROBABILITY WEIGHTING FUNCTIONS}

Probability weighting functions have many applications in science and engineering. In this paper, we are most concerned with using them to re-weight the probabilities of outcomes. Weighting is suggested by Cumulative Prospect Theory (CPT) as an important part of the human decision making process. Prospect Theory (PT), the predecessor to CPT, was suggested in the 1970s by Kahneman and Tversky (1979). They were unsatisfied with PT and suggested its improved version, CPT, in the 1990s Tversky and Kahneman (1992). CPT improves PT by re-weighting the outcome cumulative probability function instead of the outcome probability density function. This elegant approach can also be useful for global optimization problems. The purpose of this section is to familiarize the reader with probability weighting functions, which will be used later for iteratively updating probability weights. We first introduce several definitions to assist us in our discussion.

Definition 1 A weighting function, $w:[0,1] \rightarrow[0,1]$, is a monotonically increasing and Lipschitz continuous function with $w(0)=0$ and $w(1)=1$.

Definition 2 A weighting function, $w:[0,1] \rightarrow[0,1]$, is optimal-seeking if

$$
w(\alpha x+(1-\alpha) y)>\alpha w(x)+(1-\alpha) w(y), \forall \alpha \in(0,1), x \neq y \in[0,1] .
$$

Optimal-seeking is called risk-seeking in fields that model risk-sensitivity. 


\section{Lin and Marcus}

Assumption $2 w$ is an optimal-seeking weighting function.

Examples of weighting functions can be found in Diecidue et al. (2009). An optimal-seeking weighting function tends to place more weight on highly unlikely, yet highly rewarding outcomes. In the sequel, we apply the optimal-seeking weighting functions to good-news functions (i.e., complementary cumulative distribution functions). It should be noted that the optimal-seeking re-weighted expected payoff is greater than that of the risk-neutral. This will be an important feature in proving the convergence of the CWO method.

\section{DISCRETE SOLUTION SPACE}

We want to find a solution for Equation (2) assuming that

$$
\mathscr{X}:=\{1,2,3,4, \ldots\} .
$$

We further assume the discrete topology for $\mathscr{X}$. We denote the set of optimal measures on $(\mathscr{X}, \mathscr{B}(\mathscr{X}))$ by

$$
\mathscr{P}_{\mathscr{X}}^{*}:=\left\{\mathbb{P} \in \mathscr{P}_{\mathscr{X}} \mid \mathbb{P}\left(\mathscr{X}^{*}\right)=1\right\},
$$

where $\mathscr{X}^{*}$ is the set of all optimal solutions, i.e.,

$$
\mathscr{X}^{*}:=\left\{i^{*} \in \mathscr{X} \mid H(i) \leq H\left(i^{*}\right) \forall i \in \mathscr{X}\right\},
$$

and $\mathscr{P}_{\mathscr{X}}$ is the set of all possible probability measures over $\mathscr{B}(\mathscr{X})$. It should not surprise the reader that if we can find an element of $\mathscr{P}_{\mathscr{X}}^{*}$, then we have found a solution to the global optimization problem stated in Equation (2). We assume $\mathscr{X}^{*}$ has only a finite number of elements.

Assumption 3 The objective function, $H: \mathscr{X} \rightarrow \mathscr{R}$, has a finite number of optimal solutions, i.e., the set

$$
\mathscr{X}^{*}:=\left\{i^{*} \in \mathscr{X} \mid H(i) \leq H\left(i^{*}\right) \forall i \in \mathscr{X}\right\}
$$

has a finite number of elements.

Our objective is to restrict the temporal evolution of the probability measure such that it will eventually concentrate its probability density at the optimal solutions. This evolution can be defined on a measurable space, $\left(\mathscr{X} \times \mathscr{R}^{+}, \mathscr{B}\left(\mathscr{X} \times \mathscr{R}^{+}\right)\right)$, where $\mathscr{X}$ is the given solution space and $\mathscr{R}^{+}$represents time. If the evolution happens in discrete time or iteration steps, then $\mathscr{R}^{+}$can be replaced by $\{0,1,2,3, \ldots\}$. To solve Equation (1), we want to find a probability measure $\mathbb{P}$ and a $t^{*} \in \mathscr{R}^{+}$such that

$$
\mathbb{P}\left(\left\{(t, i) \mid i \in \mathscr{X}^{*}\right\}\right)=\mathbb{P}(\{(t, i) \mid i \in \mathscr{X}\}), \forall t \geq t^{*} .
$$

In other words, $\mathbb{P}$ at some finite time $t^{*}$ is a member of $\mathscr{P}_{\mathscr{X}}^{*}$. We denote the resulting probability space by

$$
\left(\mathscr{X} \times \mathscr{R}^{+}, \mathscr{B}\left(\mathscr{X} \times \mathscr{R}^{+}\right), \mathbb{P}\right) .
$$

At each time $t, \mathbb{P}$ induces a probability measure on the measurable space $(\mathscr{X}, \mathscr{B}(\mathscr{X}))$,

$$
\mathbb{P}_{t}\left(B_{\mathscr{X}}\right):=\mathbb{P}\left(\left\{(t, i) \mid i \in B_{\mathscr{X}}\right\}\right), \forall B_{\mathscr{X}} \in \mathscr{B}(\mathscr{X}),
$$

resulting in a probability space $\left(\mathscr{X}, \mathscr{B}(\mathscr{X}), \mathbb{P}_{t}\right)$. Conversely, if we know $\mathbb{P}_{t}$ at all times, then we can construct a $\mathbb{P}$ that satisfies Equation (3). The coordinate random variable is denoted by $X$ (i.e., $X(i)=i, i \in \mathscr{X}$ ). Similarly, the outcome random variable is denoted by $Y$, where $Y=\phi(H(X))$. We denote the set of all possible outcomes from evaluating $\phi(H(\cdot))$ over $\mathscr{X}$ by

$$
\mathscr{Y}:=\left\{y \in \mathscr{R}^{+} \mid \exists i \in \mathscr{X} \text { s.t. } y=\phi(H(i))\right\} .
$$




\section{Lin and Marcus}

A sensible next step is to write down the dynamics of $\mathbb{P}_{t}$ with respect to time (i.e., $\dot{\mathbb{P}}_{t}$ ), and interestingly we will be able to prove that

$$
\mathbb{E}_{t}[\phi(H(X))]:=\int_{\mathscr{X}} \phi(H(i)) d \mathbb{P}_{t}:=\int_{\mathscr{Y}} y d \mathbb{P}_{t}^{\phi(H(X))}
$$

is a function strictly increasing in $t$. In the equation above, $\mathbb{P}_{t}^{\phi(H(X))}$ is the probability measure of the random variable $\phi(H(X))$ induced by $X$. Of course, probability weighting functions from Section 3 play a key role in the equations for $\dot{\mathbb{P}}_{t}$ (We opted for the notation $\mathbb{P}_{t}$ instead of $\mathbb{P}_{t}^{w}$ for simplicity, but the reader should be mindful of $\mathbb{P}_{t}$ 's dependence on $w$.). Using Equation (4) along with Lyapunov stability analysis, we will conclude the convergence of $\mathbb{P}_{t}$ to an optimal solution (i.e., an element of $\mathscr{P}_{\mathscr{X}}^{*}$ ).

The generalized solution probability vector equation has the form:

$$
\begin{aligned}
\frac{d x_{i}(t)}{d t}= & \beta_{i}(t)\left(w\left(\sum_{j: \phi(H(j)) \geq \phi(H(i))} x_{j}(t)\right)-w\left(\sum_{j: \phi(H(j))>\phi(H(i))} x_{j}(t)\right)\right) \\
& \cdots-x_{i}(t) \forall i \in \mathscr{X} \\
& \sum_{\phi(H(i))=y} \beta(i, y, t)=1, \forall y \in \mathscr{Y} \forall t \in \mathscr{R}^{+},
\end{aligned}
$$

where $x_{i}: \mathscr{R}^{+} \rightarrow[0,1]$ is the probability measure assigned to an element $i \in \mathscr{X}$, and $\beta_{i}(t):=\beta(i, \phi(H(i)), t)$ is a distribution rule defined below. In Equation (5), which is a nonlinear Fokker-Plank equation (cf. Kolokoltsov (2010), Frank (2005)), the difference between the first $w$ distorted term and the second $w$ distorted term is the event $\phi(H(j))=\phi(H(i))$. Wang et al. (2010) have an alternative set of evolution equations, also nonlinear Fokker-Plank equations, motivated by evolutionary game theory.

Definition 3 A distribution rule with respect to a given objective function, $\phi(H(\cdot))$, is a mapping $\beta$ : $\mathscr{X} \times \mathscr{Y} \times \mathscr{R}^{+} \rightarrow[0,1]$ such that

$$
\sum_{\phi(H(i))=y} \beta(i, y, t) d x=1 \forall y \in \mathscr{Y} \forall t \in \mathscr{R}^{+} .
$$

Connecting this equation with the discussion at the beginning of this section, the reader should note that

$$
\mathbb{P}_{t}(X=i)=x_{i}(t) \forall i \in \mathscr{X} \text {. }
$$

The generalized outcome probability vector equation has the form:

$$
\frac{d y_{z}(t)}{d t}=w\left(\sum_{j: j \geq z} y_{j}(t)\right)-w\left(\sum_{j: j>z} y_{j}(t)\right)-y_{z}(t) \forall z \in \mathscr{Y} .
$$

We pay special attention to the best outcome equation:

$$
\frac{d y_{*}(t)}{d t}=w\left(\sum_{j: j \geq *} y_{j}(t)\right)-y_{*}(t)
$$

where $*:=\phi\left(H\left(i^{*}\right)\right) i^{*} \in \mathscr{X}^{*}$.

In the rest of this section, we want to study the convergence properties of Equation (5). Furthermore, we want to understand the stability properties, in the Lyapunov sense, of its limit points. The first step in understanding Equation (5) is to understand the existence and uniqueness of its solutions. The outline of our proof follows Oechssler and Riedel (2001) and Hofbauer et al. (2009). 


\section{Lin and Marcus}

Theorem 4 For each $x(0) \in \mathscr{P}_{\mathscr{X}}$, the ordinary differential equation (5) has a unique solution for $t \in \mathscr{R}^{+}$. Here, $\beta: \mathscr{X} \times \mathscr{Y} \times \mathscr{R}^{+} \rightarrow[0,1]$ is a distribution rule.

Proof. For the proof of this theorem, we are only using the fact that $\beta$ is a bounded function. In the future, $\beta$ could depend on both $i \in \mathscr{X}$ and $x(t) \in \mathscr{P}_{\mathscr{X}}$. We use the total variation norm, $\|\cdot\|$, on a $\sigma$-finite signed measure space over $(\mathscr{X}, \mathscr{B}(\mathscr{X}))$ :

$$
\|x(t)\|=\sup _{A \in \mathscr{B}(\mathscr{X})} \sum_{i \in A}\left|x_{i}(t)\right| .
$$

Since $x(t) \in \mathscr{P}_{\mathscr{X}}$ is a probability measure $\forall t$, and $\left|\beta_{i}\right| \leq 1$, we know the following inequalities hold:

$$
\begin{gathered}
\sup _{A \in \mathscr{B}(\mathscr{X})} \sum_{i \in A}\left|\beta_{i}(t)\left(w\left(\sum_{j: \phi(H(j)) \geq \phi(H(i))} x_{j}(t)\right)-w\left(\sum_{j: \phi(H(j))>\phi(H(i))} x_{j}(t)\right)\right)-x_{i}(t)\right| \\
\leq \quad \sup _{A \in \mathscr{B}(\mathscr{X})} \sum_{i \in A}\left|\beta_{i}(t)\left(w\left(\sum_{j: \phi(H(j)) \geq \phi(H(i))} x_{j}(t)\right)-w\left(\sum_{j: \phi(H(j))>\phi(H(i))} x_{j}(t)\right)\right)\right| \\
+\sup _{A \in \mathscr{B}(\mathscr{X})} \sum_{i \in A}\left|x_{i}(t)\right| \leq 2 .
\end{gathered}
$$

Hence, we conclude that the right hand side of Equation (5) is bounded by 2. Next, we need to prove that the right hand side of Equation (5) is Lipschitz continuous.

$$
\begin{aligned}
& \sup _{A \in \mathscr{B}(\mathscr{X})} \sum_{i \in A} \mid\left(\beta_{i}(t)\left(w\left(\sum_{j: \phi(H(j)) \geq \phi(H(i))} x_{j}^{1}(t)\right)-w\left(\sum_{j: \phi(H(j))>\phi(H(i))} x_{j}^{1}(t)\right)\right)-x_{i}^{1}(t)\right) \\
& \leq \sup _{A \in \mathscr{B}(\mathscr{X})} \sum_{i \in A} \mid\left(w\left(\sum_{j: \phi(H(j)) \geq \phi(H(i))} x_{j}^{1}(t)\right)-w\left(\sum_{j: \phi(H(j))>\phi(H(i))} x_{j}^{1}(t)\right)\right)-x_{i}^{1}(t) \\
& -\left(\left(w\left(\sum_{j: \phi(H(j)) \geq \phi(H(i))} x_{j}^{2}(t)\right)-w\left(\sum_{j: \phi(H(j))>\phi(H(i))} x_{j}^{2}(t)\right)\right)-x_{i}^{2}(t)\right) \mid \\
& \leq \sup _{A \in \mathscr{B}(\mathscr{X})} \sum_{i \in A} \mid w\left(\sum_{j: \phi(H(j)) \geq \phi(H(i))} x_{j}^{1}(t)\right)-w\left(\sum_{j: \phi(H(j))>\phi(H(i))} x_{j}^{1}(t)\right) \\
& -\left(w\left(\sum_{j: \phi(H(j)) \geq \phi(H(i))} x_{j}^{2}(t)\right)-w\left(\sum_{j: \phi(H(j))>\phi(H(i))} x_{j}^{2}(t)\right)\right)|+| x_{i}^{1}(t)-x_{i}^{2}(t) \mid \\
& \leq K \sup _{A \in \mathscr{B}(\mathscr{X})} \sum_{i \in A}\left|\sum_{j: \phi(H(j))=\phi(H(i))} x_{j}^{1}(t)-\sum_{j: \phi(H(j))=\phi(H(i))} x_{j}^{2}(t)\right| \\
& +\sup _{A \in \mathscr{B}(\mathscr{X})} \sum_{i \in A}\left|x_{i}^{1}(t)-x_{i}^{2}(t)\right| \\
& \leq K \sup _{A \in \mathscr{B}(\mathscr{X})} \sum_{i \in A}\left|\sum_{j: \phi(H(j))=\phi(H(i))} x_{j}^{1}(t)-x_{j}^{2}(t)\right|+\sup _{A \in \mathscr{B}(\mathscr{X})} \sum_{i \in A}\left|x_{i}^{1}(t)-x_{i}^{2}(t)\right| \\
& \leq K\left\|x^{1}(t)-x^{2}(t)\right\|+\left\|x^{1}(t)-x^{2}(t)\right\| \leq(K+1)\left\|x^{1}(t)-x^{2}(t)\right\| .
\end{aligned}
$$

Hence, the right hand side of Equation (5) is Lipschitz continuous in $x$ with the constant $\mathrm{K}+1$, where $\mathrm{K}$ is the Lipschitz constant for $w$ (see Definition 1). Using Corollary 3.9 of Zeidler (1989), we conclude that Equation (5) with an initial measure $x(0) \in \mathscr{P}_{\mathscr{X}}$ has a unique solution $x(t) \forall t \in \mathscr{R}^{+}$.

The next Lemma is needed in Theorem 8, which shows $\mathbb{E}_{t}[\phi(H(X))]$ is monotonically increasing in $t$.

Lemma 5 Given an optimal-seeking weighting function, $w$, there exists a $\tilde{\zeta}$ such that

$$
\sum_{\zeta \in \mathscr{Y}} \zeta\left(w\left(\sum_{j: Y \geq \zeta} y_{j}(t)\right)-w\left(\sum_{j: Y>\zeta} y_{j}(t)\right)-y_{\zeta}(t)\right)
$$




\section{Lin and Marcus}

can be decomposed into the sum of its non-negative and negative parts:

$$
\underbrace{\sum_{\zeta \geq \tilde{\zeta}} \zeta\left(w\left(\sum_{j: Y \geq \zeta} y_{j}(t)\right)-w\left(\sum_{j: Y>\zeta} y_{j}(t)\right)-y_{\zeta}(t)\right)}_{\text {non-negative }} .
$$

Proof. Since $w$ is a monotonically increasing function, it satisfies

$$
\frac{w\left(\sum_{j: Y \geq \zeta} y_{j}(t)\right)-w\left(\sum_{j: Y>\zeta} y_{j}(t)\right)}{y_{\zeta}(t)} \geq 0 .
$$

Furthermore, since $w$ is an optimal-seeking function we have

$$
\begin{array}{r}
\frac{w\left(\sum_{j: Y \geq \zeta_{1}} y_{j}(t)\right)-w\left(\sum_{j: Y>\zeta_{1}} y_{j}(t)\right)}{y_{\zeta_{1}}(t)}>\frac{w\left(\sum_{j: Y \geq \zeta_{2}} y_{j}(t)\right)-w\left(\sum_{j: Y>\zeta_{2}} y_{j}(t)\right)}{y_{\zeta_{2}}(t)} \\
\forall \zeta_{1} \geq \zeta_{2} \in \mathscr{Y} .
\end{array}
$$

In addition, since $w(0)=0$ and $w(1)=1$, we know that

$$
\frac{w\left(\sum_{j: Y \geq \zeta} y_{j}(t)\right)-w\left(\sum_{j: Y>\zeta} y_{j}(t)\right)}{y_{\zeta}(t)}>1
$$

for some $\zeta \in \mathscr{Y}$. From Equation (6) we know if $\zeta_{2}$ satisfies the above inequality, then so does $\zeta_{1} \geq \zeta_{2} \in \mathscr{Y}$. Hence, we can conclude that $\tilde{\zeta}$ is the smallest such $\zeta$.

At the beginning of this section, we stated implicitly that if we can find an element of $\mathscr{P}_{\mathscr{X}}^{*}$, then we have found a solution to the global optimization problem stated in Equation (2). The theorems below present a blueprint, through the use of Equation (5), to obtain an element of $\mathscr{P}_{\mathscr{X}}^{*}$. In Theorem 4, an initial point can be any element of $\mathscr{P}_{\mathscr{X}}$. As we have discovered, $\mathscr{P}_{\mathscr{X}}$ is too large a set to initialize Equation (5) to guarantee as $t \rightarrow \infty$ the solution probability vector, $x(t)$, will be an element of $\mathscr{P}_{\mathscr{X}}^{*}$. Hence, we need to constrain our initial points to a smaller set.

Definition 4 We denote the set of all $x(0)$ for which there exists an optimal solution, $i^{*} \in \mathscr{X}^{*}$, such that $x_{i^{*}}(0)>0$ by $\mathscr{O}$.

In other words, $\mathscr{O}$ contains all initial probability vectors with nonzero weights on at least one optimal solution. The next theorem proves the total probability measure on the optimal solution set will converge to 1 as $t \rightarrow \infty$. On the other hand, the total probability measure on the non-optimal solution set will converge to 0 as $t \rightarrow \infty$.

Theorem 6 If $x(t)$ is a solution for Equation (5), then it satisfies the following with $x(0) \in \mathscr{O}$ :

1) The total probability weight on the optimal solutions, $\sum_{i \in \mathscr{X}^{*}} x_{i}(t)$, is a monotonically increasing function of $\mathrm{t}$. In fact, it converges to 1 as $t \rightarrow \infty$;

2) The probability of any non-optimal solution, $x_{i}(t): \mathscr{R}^{+} \rightarrow[0,1] i \notin \mathscr{X}^{*}$, approaches zero as $\mathrm{t} \rightarrow \infty$. 


\section{Lin and Marcus}

Proof. We know that

$$
\sum_{i \in \mathscr{X}^{*}} x_{i}(t)=y_{*}(t)
$$

hence we only need to prove $y_{*}$ is a monotonically increasing function of $\mathrm{t}$. By writing down the equation for $y_{*}$ :

$$
\frac{d y_{*}(t)}{d t}=w\left(\sum_{j: j \geq *} y_{j}(t)\right)-y_{*}(t),
$$

and knowing the optimal-seeking property of $w$

$$
w\left(\sum_{j: j \geq *} y_{j}(t)\right)>y_{*}(t),
$$

we conclude that

$$
\frac{d y_{*}(t)}{d t}>0 \forall y_{*}(t) \neq 1 \text {, and } \frac{d y_{*}(t)}{d t}=0 \text { when } y_{*}(t)=1 \text {. }
$$

Since $x(0) \in \mathscr{O}$ implies $y_{*}(0)>0$, the first claim is proved.

The second claim follows from the first claim. Since $y_{*}(\infty)=1$, and $x$ is a solution probability vector (i.e., sum of the $x_{i}$ s is 1 ), we can conclude the following:

$$
\begin{aligned}
\lim _{t \rightarrow \infty} \sum_{i \in \mathscr{X}} x_{i}(t) & =\lim _{t \rightarrow \infty} \sum_{i \in \mathscr{X}^{*}} x_{i}(t)+\sum_{i \notin \mathscr{X}^{*}} x_{i}(t)=1+\lim _{t \rightarrow \infty} \sum_{i \notin \mathscr{X}^{*}} x_{i}(t)=1 \\
\Longrightarrow & \lim _{t \rightarrow \infty} \sum_{i \notin \mathscr{X}^{*}} x_{i}(t)=0 \Longrightarrow \lim _{t \rightarrow \infty} x_{i}(t)=0 \forall i \notin \mathscr{X}^{*} .
\end{aligned}
$$

The conclusion follows.

We are interested in finding the limit points of Equation (5). Ideally, these limit points should be elements in $\mathscr{P}_{\mathscr{X}}^{*}$. This is accomplished by picking the initial point set more carefully.

Definition 5 The limit set of Equation (5), with all distribution rules, starting from an element $x(0) \in \mathscr{I}$ is

$$
\mathscr{E} \mathscr{I}:=\left\{x_{\infty} \in \mathscr{P}_{\mathscr{X}} \mid x_{\infty}=\lim _{t \rightarrow \infty} x(t), x(0) \in \mathscr{I}\right\} .
$$

We characterize the limit set of Equation (5) when $x(0) \in \mathscr{O}$ in the following theorem.

Theorem 7 The limit set of Equation (5) started in $\mathscr{O}$ is $\mathscr{P}_{\mathscr{X}}^{*}$, i.e.,

$$
\mathscr{E}_{\mathscr{O}}=\mathscr{P}_{\mathscr{X}}^{*}:=\left\{x \in \mathscr{P}_{\mathscr{X}} \mid \sum_{i^{*} \in \mathscr{K}^{*}} x_{i^{*}}=1\right\} .
$$

Proof. $\quad$ To prove the first claim, we will first prove $\mathscr{E}_{\mathscr{O}} \supset \mathscr{P}_{\mathscr{X}}^{*}$, then we will prove $\mathscr{E}_{\mathscr{O}} \subset \mathscr{P}_{\mathscr{X}}^{*}$. The first case, $\mathscr{E}_{\mathscr{O}} \supset \mathscr{P}_{\mathscr{X}}^{*}$, can be trivially proved by taking an element $x \in \mathscr{P}_{\mathscr{X}}^{*}$, we notice that $x \in \mathscr{O}$, and by definition of $\mathscr{E}_{\mathscr{O}}$ (i.e., the limit set of Equation (5) staring from $\mathscr{O}$ ), we conclude $x \in \mathscr{E}_{\mathscr{O}}$.

Now we proceed to prove $\mathscr{E}_{\mathscr{O}} \subset \mathscr{P}_{\mathscr{X}}^{*}$. We prove by contradiction. Assume there is an element $e \in \mathscr{E}_{\mathscr{O}}$, but not in $\mathscr{P}_{\mathscr{X}}^{*}$ such that:

$$
\begin{aligned}
\dot{e}_{i}(t)= & \beta_{i}(t)\left(w\left(\sum_{j: \phi(H(j)) \geq \phi(H(i))} e_{j}(t)\right)-w\left(\sum_{j: \phi(H(j))>\phi(H(i))} e_{j}(t)\right)\right)-e_{i}(t) \\
& e_{i}(0) \geq 0, e_{i}(\infty)>0, i \notin \mathscr{X}^{*} .
\end{aligned}
$$

This contradicts the second claim of Theorem 6 , where $e_{i}(\infty)=0$. 


\section{Lin and Marcus}

The next theorem shows the monotonically increasing nature of $\mathbb{E}_{t}[\phi(H(X))]$, which will be useful later in proving some stability properties for Equation (5).

Theorem 8 Let $x(t)$ be a solution of the dynamics represented by Equation (5) with an initial point in $\mathscr{O}$. Then the following statements are true:

1) The expected outcome, i.e., $\mathbb{E}_{t}[\phi(H(X))]:=\sum_{i \in \mathscr{X}} \phi(H(i)) x_{i}(t)$, is monotonically increasing with $t$

2) If $x(t) \notin \mathscr{E}_{\mathscr{O}}$ for any $t \in \mathscr{R}^{+}$, then $\mathbb{E}_{t}[\phi(H(X))]$ is strictly increasing with $t$.

Proof. We start our proof by differentiating the average outcome function:

$$
\begin{aligned}
\frac{d}{d t} \mathbb{E}_{t}[\phi(H(X))] & =\frac{d}{d t} \mathbb{E}_{t}[Y] \\
& =\sum_{\zeta \geq \tilde{\zeta}} \zeta\left(w\left(\sum_{j: Y \geq \zeta} y_{j}(t)\right)-w\left(\sum_{j: Y>\zeta} y_{j}(t)\right)-y_{\zeta}(t)\right) \\
& +\sum_{\zeta<\tilde{\zeta}} \zeta\left(w\left(\sum_{j: Y \geq \zeta} y_{j}(t)\right)-w\left(\sum_{j: Y>\zeta} y_{j}(t)\right)-y_{\zeta}(t)\right) \text { (Lemma. 5) } \\
& \geq \tilde{\zeta}\left(\sum_{\zeta \in \mathscr{Y}} w\left(\sum_{j: Y \geq \zeta} y_{j}(t)\right)-w\left(\sum_{j: Y>\zeta} y_{j}(t)\right)-y_{\zeta}(t)\right) \\
& =\tilde{\zeta} \times 0=0 .
\end{aligned}
$$

Here, the last equality is true because

$$
\sum_{\zeta \in \mathscr{Y}} w\left(\sum_{j: Y \geq \zeta} y_{j}(t)\right)-w\left(\sum_{j: Y>\zeta} y_{j}(t)\right)=1 \text {, and } \sum_{\zeta \in \mathscr{Y}} y_{\zeta}(t)=1 .
$$

The $\tilde{\zeta}$ variable is used to decompose the expected outcome function into non-negative and negative parts (see Lemma 5). The first claim is proved.

The second claim is proved by contradiction. We assume that $x(t)$ is not in the limit set, and

$$
\frac{d}{d t} \mathbb{E}_{t}[\phi(H(X))]=0
$$

Along with Theorem 6 , the equality above implies that $x(t)$, the density function at time $t$, has all its probability mass on the optimal solutions. From Theorem 7, we know a limit point has its probability mass on the optimal solutions. However, we assumed $x(t)$ is not a limit point, hence we reach a contradiction.

We will now proceed to prove some stability properties concerning Equation (5), but first we need to introduce our definitions of stability given a metric $d$.

Definition 6 Let $\mathscr{L}$ be a subset of $\mathscr{P}_{\mathscr{X}}$. For a point $x(t) \in \mathscr{P}_{\mathscr{X}}$, we define the distance between $x(t)$ and $\mathscr{L}$ as

$$
d(x(t), \mathscr{L}):=\inf \{d(x(t), q), \forall q \in \mathscr{L}\} .
$$

$\mathscr{L}$ is called Lyapunov stable if for all $\varepsilon>0$, there exists a $\delta>0$ such that

$$
d(x(0), \mathscr{L})<\delta \Rightarrow d(x(t), \mathscr{L})<\varepsilon, \forall t>0 .
$$




\section{Lin and Marcus}

Lyapunov was also interested in other stronger types of stability.

Definition 7 Let $\mathscr{L}$ be a subset of $\mathscr{P}_{\mathscr{X}} \cdot \mathscr{L}$ is called asymptotically stable if $\mathscr{L}$ is Lyapunov stable, and there exists a $\delta>0$ such that

$$
d(x(0), \mathscr{L})<\delta \Rightarrow d(x(t), \mathscr{L}) \rightarrow 0
$$

as $t \rightarrow \infty$.

The next theorem is the main result of this section. It states $\mathscr{E}_{\mathscr{O}}$ is compact and asymptotically stable.

Theorem $9 \mathscr{E}_{\mathscr{O}}$ is a compact set and it is asymptotically stable.

Proof. We need to first prove that $\mathscr{E}_{\mathscr{O}}$ is a compact set. Since from our Theorem 7, we have $\mathscr{E}_{\mathscr{O}}=\mathscr{P}_{\mathscr{X}}^{*}$ and can instead prove

$$
\mathscr{P}_{\mathscr{X}}^{*}:=\left\{x \in \mathscr{P}_{\mathscr{X}} \mid \sum_{i^{*} \in \mathscr{X}^{*}} x_{i^{*}}=1\right\}
$$

is compact. It is easy to see that $\mathscr{P}_{\mathscr{X}}^{*}$ is tight due to Assumption 3 . Furthermore, we can prove it is a closed set by contradiction. Assume there exists a sequence $\left\{x^{n}\right\} \in \mathscr{P}_{\mathscr{X}}^{*}$ such that $x^{n} \rightarrow \hat{x} \notin \mathscr{P}_{\mathscr{X}}^{*}$. This implies $\exists N$ such that $\forall n>N$ we have $\sum_{i^{*} \in \mathscr{X}^{*}} x_{i^{*}}^{n}<1$, and $\sum_{i \notin \mathscr{X}^{*}} x_{i}^{n}>0$, which contradicts the second claim of Theorem 6 . Hence, $\mathscr{P}_{\mathscr{X}}^{*}=\mathscr{E}_{\mathscr{O}}$ is a compact set. Consider the Lyapunov function

$$
V\left(x_{t}\right)=\mathbb{E}\left[\phi\left(H\left(X^{*}\right)\right)\right]-\mathbb{E}_{t}[\phi(H(X))],
$$

where $x^{*} \in \mathscr{P}_{\mathscr{O}}^{*}$ and $X^{*}$ is the corresponding random variable. Note that $V\left(x_{t}\right)$ is positive for all $x_{t} \in$ $\mathscr{P}_{\mathscr{X}} \backslash \mathscr{P}_{\mathscr{X}}^{*}$, and $V\left(x_{t}\right)=0$ for $x_{t} \in \mathscr{P}_{\mathscr{X}}^{*}=\mathscr{E}_{\mathscr{O}}$. From Theorem 8 we have $\dot{V}\left(x_{t}\right)<0$ for all $t>0$ and $x_{t} \notin \mathscr{P}_{\mathscr{O}}^{*}$. Furthermore, we know $\mathscr{E}_{\mathscr{O}}$ is a compact set. Applying a generalized version of Lyapunov's theorem (see Bhatia and Szegö (1970), Theorem 2.2), the desired conclusion is reached.

Chapter V of Bhatia and Szegö (1970) presented a generalized version of Lyapunov's theorem on a general metric space. In the proof of Theorem 9, we applied this generalized Lyapunov's theorem on the Banach Space of $\sigma$-finite signed measures over $(\mathscr{X}, \mathscr{B}(\mathscr{X}))$, where we used the total variation distance

$$
d\left(x^{1}, x^{2}\right)=\sup _{A \in \mathscr{B}(\mathscr{X})} \sum_{j \in A}\left|x_{j}^{1}(t)-x_{j}^{2}(t)\right| .
$$

We have proven so far that if we start Equation (5) in $\mathscr{O}$, then the possible limit points are elements of $\mathscr{E}_{\mathscr{O}}=\mathscr{P}_{\mathscr{X}}^{*}$. In other words, the limit points are the set of optimal solution probability vectors. Furthermore, the set $\mathscr{E}_{\mathscr{O}}$ is asymptomatically stable.

\section{NUMERICAL EXAMPLES: ASYMMETRIC TRAVELING SALESMAN PROBLEMS (ATSP)}

We modify the model-based algorithm found in $\mathrm{Hu}$ et al. (2007) by changing how the density is propagated. The modified algorithm (see Algorithm 1) is applied to an asymmetric traveling salesman problem taken from the website http://www.iwr.uni-heidelberg.de/groups/comopt/software/TSPLIB95. When step 5 of Algorithm 1, the weight-update stage, is applied assuming uniform sample weight and the weighting function

$$
w(p):=\frac{10 p \sigma+\ln \left(1+e^{-\sigma}\right)-\ln \left(1+e^{(-1+10 p) \sigma}\right)}{10 \theta+\ln \left(1+e^{-\rho}\right)-\ln \left(1+e^{9 \sigma}\right)},
$$




\section{Lin and Marcus}

where $\sigma$ is the optimal-seeking factor and $\rho$ is the quantile threshold, the resulting algorithm is named CWO_U. Algorithm 1 will converge under Equation (5) using any optimal-seeking $w$. The choice of Equation (7) as the weighting function $w$ is due to its connection to $\mathrm{CE}$, which is explained below.

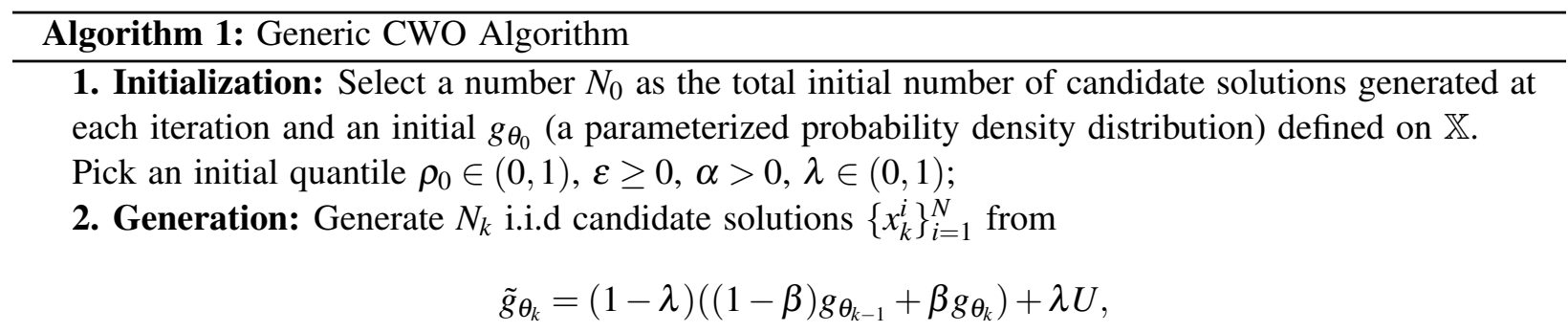

where $U$ is the uniform distribution;

3. Quantile-Update: Calculate the $\left(1-\rho_{k}\right)$-quantile, $\tilde{\gamma}_{k+1}\left(\rho_{k}, N_{k}\right):=\phi(H)_{\left(\left\lceil\left(1-\rho_{k}\right) N_{k}\right\rceil\right)}$, where $\lceil a\rceil$ is the smallest integer greater than a and $H_{(i)}$ is the $\mathrm{i}$-th highest value for the sequence $\left\{\phi\left(H\left(x_{k}^{i}\right)\right)\right\}_{i=1}^{N_{k}}$;

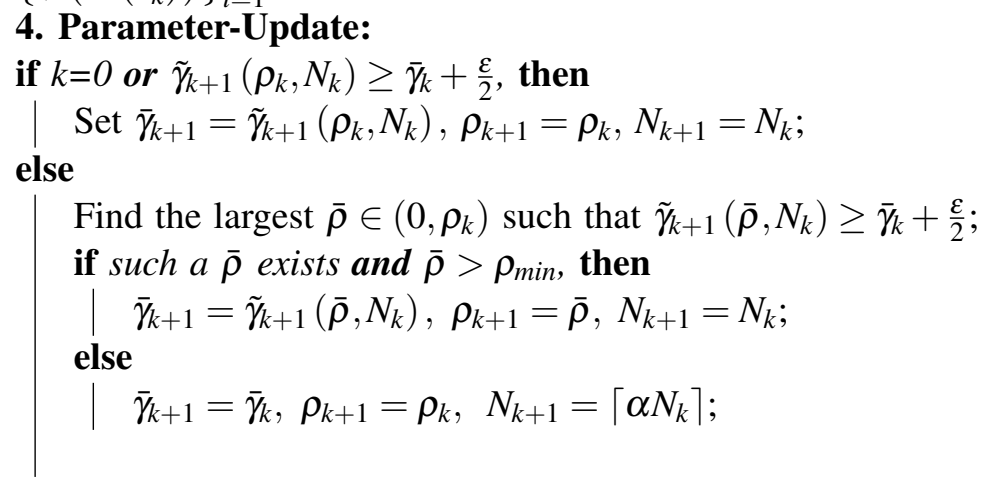

5. Weight-Update: Update the weights of the generated samples $\left\{x_{k}^{i}\right\}_{i=1}^{N}$ according to weight updating methods based on Equation (5), producing the p.m.f $p_{X, k+1}=\sum_{i=1}^{N} w_{k+1}^{i} \delta\left(x-x_{k}^{i}\right)$, where $w_{k+1}^{i}$ is the updated weight for $x_{k}^{i}$;

6. Density Projection: Construct $g_{\theta_{k+1}}$ by projecting the density $p_{X, k+1}=\sum_{i=1}^{N} w_{k+1}^{i} \delta\left(x-x_{k}^{i}\right)$ onto $g_{\theta}$ by solving the equation:

$$
\theta_{k+1}=\arg \max _{\theta \in \Theta} \sum_{i=1}^{N} w_{k+1}^{i} \ln g_{\theta}\left(x_{k}^{i}\right) ;
$$

7. Stop: if some stopping criterion is satisfied; otherwise go to step 2 and $k=k+1$.

We remind the reader that the density update equation for cross entropy is

$$
\begin{aligned}
p_{X, k+1}^{C E}(x) & =\frac{1\{\phi(H(s))>\gamma\}}{l} p_{X, k}^{C E}(x) \\
& \propto 1\{\phi(H(s))>\gamma\} p_{X, k}^{C E}(x),
\end{aligned}
$$

where an indicator function is used to select the elite samples. In fact, the cross-entropy equation is just the limiting case, as $\sigma \rightarrow \infty$ in Equation (7), of the CWO_U algorithm. For each iteration, we increase the value of $\sigma$ proportional to the iteration count (i.e., $\sigma=\Delta k$ ). As we increase the optimal-seeking factor, the derivative of Equation (7) will approach a step function (i.e., Equation (8)) with its discontinuity occurring at $\rho=0.1$. 


\section{Lin and Marcus}

We will compare the performance of our CWO_U algorithm against that of the CE algorithm. In the following numerical examples, we fixed $\Delta$ to be 0.01 . Table 1 contains the results from running 20 trials of CWO_U and CE algorithms with the parameters $\rho_{0}=0.1, \rho_{\min }=0.001, N_{0}=1000, \varepsilon=0, \alpha=1$, $\lambda=0.01$, and $\beta=0.7$.

Table 1: CWO_U and CE performance results.

\begin{tabular}{|c|c|c|c|c|c|c|c|c|}
\hline ATSP & $N_{\text {cities }}$ & $N_{\text {Total }}($ Std. err. $)$ & $H_{\text {best }}$ & $H_{*}$ & $H^{*}$ & $\delta_{*}$ & $\delta^{*}$ & $\delta$ (Std. err. $)$ \\
\hline \hline $\mathrm{ft} 53$ & 53 & $90,450(6.0 \mathrm{e} 3)$ & 6,905 & 7,679 & 7,037 & 0.112 & 0.0191 & $0.060(0.0244)$ \\
\hline ce_ft53 & 53 & $65,100(5.7 \mathrm{e} 3)$ & 6,905 & 7,676 & 7,088 & 0.111 & 0.0265 & $0.075(0.0276)$ \\
\hline
\end{tabular}

\section{CONCLUSION}

In the first part of this paper, we proved the convergence of CWO-based algorithms. The proofs provide a rigorous mathematical foundation for the $\mathrm{CWO}_{-} \mathrm{U}$ algorithm we proposed in the numerical examples section. Interestingly, the standard cross-entropy (CE) approach is just a limiting case of the CWO_U algorithm. Comparing the numerical results of CWO_U with those of CE, we believe our algorithm is better at obtaining an optimal solution. Of course, the improvement in performance is at the expense of increasing computational costs.

\section{ACKNOWLEDGMENTS}

This work was supported in part by the National Science Foundation (NSF) under Grants CNS-0926194, CMMI-0856256, CMMI-0900332, and EECS-0901543, and by the Air Force Office of Scientific Research (AFOSR) under Grant FA9550-10-1-0340.

\section{REFERENCES}

Bhatia, N. P., and G. P. Szegö. 1970. Stability Theory of Dynamical Systems. New York: Springer-Verlag. Diecidue, E., U. Schmidt, and H. Zank. 2009. "Parametric Weighting Functions". Journal of Economic Theory 144 (3): 1102-1118.

Frank, T. D. 2005. Nonlinear Fokker-Planck Equations - Fundamentals and Applications. Springer-Verlag.

Hofbauer, J., J. Oechssler, and F. Riedel. 2009. "Brown-von Neumann-Nash Dynamics: The Continuous Strategy Case". Games and Economic Behavior 65 (2): 406-429.

Hu, J., M. C. Fu, and S. I. Marcus. 2007. "A Model Reference Adaptive Search Method for Global Optimization". Operations Research 55 (3): 549-568.

Hu, J., Y. Wang, E. Zhou, M. C. Fu, and S. I. Marcus. 2012. "A Survey of Some Model-Based Methods for Global Optimization". In Optimization, Control, and Applications of Stochastic Systems, edited by D. Hernández-Hernández and J. A. Minjárez-Sosa, 157-179. Boston: Birkhäuser Boston.

Kahneman, D., and A. Tversky. 1979. Prospect Theory: An Analysis of Decision under Risk. National Emergency Training Center.

Kolokoltsov, V. N. 2010. Nonlinear Markov Processes and Kinetic Equations. Cambridge University Press.

Oechssler, J., and F. Riedel. 2001. "Evolutionary Dynamics on Infinite Strategy Spaces”. Economic Theory 17 (1): $141-162$.

Tversky, A., and D. Kahneman. 1992. "Advances in Prospect Theory: Cumulative Representation of Uncertainty". Journal of Risk and Uncertainty 5 (4): 297-323.

Wang, Y. 2011. Simulation-Based Methods for Stochastic Control and Global Optimization. Ph. D. thesis, University of Maryland - College Park.

Wang, Y., M. C. Fu, and S. I. Marcus. 2010. "Model-based Evolutionary Optimization". In Proceedings of the Winter Simulation Conference, edited by S. J. B. Johansson, J. Montoya-Torres, J. Hugan, and E. Yücesan, 1199-1210. Piscataway, New Jersey: Institute of Electrical and Electronics Engineers, Inc. 


\section{Lin and Marcus}

Zeidler, E. 1989. Nonlinear Functional Analysis and Its Applications: Part 2 B: Nonlinear Monotone Operators. Springer.

\section{AUTHOR BIOGRAPHIES}

Kun Lin is a graduate student in the Department of Electrical \& Computer Engineering and the Institute for Systems Research, University of Maryland. His research interests are stochastic control theory and simulation optimization. His email address is klin@umd.edu.

Steven I. Marcus is a Professor in the Department of Electrical and Computer Engineering and the Institute for Systems Research, University of Maryland. He received the B.A. degree in electrical engineering and mathematic from Rice University in 1971, and the S.M. and Ph.D. degrees in electrical engineering from MIT in 1972 and 1975, respectively. From 1975 to 1991, he was with the Department of Electrical and Computer Engineering, University of Texas, Austin, where he was the L.B. (Preach) Meaders Professor in Engineering. At the University of Maryland, he has served as Director of the Institute for Systems Research from 1991-1996, and as Chair of the Electrical and Computer Engineering Department from 2000-2005. He is former Editor-in-Chief of the SIAM Journal on Control and Optimization. He is a Fellow of IEEE and SIAM. His email address is marcus@umd.edu. 